\title{
SOURCES OF AND REMEDIES FOR REMOVING UNWANTED REFLECTIONS IN MILLIMETER WAVE IMAGES OF COMPLEX SOFI-COVERED SPACE SHUTTLE STRUCTURES
}

\author{
S. Kharkovsky ${ }^{1}$, R. Zoughi ${ }^{1}$, and F.L. Hepburn ${ }^{2}$ \\ ${ }^{1}$ Electrical and Computer Engineering Department \\ Applied Microwave Nondestructive Testing Laboratory (amntt) \\ University of Missouri-Rolla \\ Rolla, MO 65409 \\ ${ }^{2}$ NASA George C. Marshall Space Flight Center \\ Marshall Space Flight Center, Huntsville, AL 35812
}

\begin{abstract}
In the recent years, continuous-wave near-field and lens-focused millimeter wave imaging systems have been effectively used to demonstrate their utility for producing high-resolution images of metallic structures covered with spay on foam insulation (SOFI) such as the Space Shuttle external fuel tank. However, for some specific structures a certain interference pattern may be superimposed on the produced images. There are methods by which the influence of this unwanted interference can be reduced, such as the incorporation of an incidence angle and the proper use of signal polarization. This paper presents the basics of this problem and describes the use of the methods for reducing this unwanted influence through specific examples.
\end{abstract}

Keywords: SOFI, Space Shuttle, Nondestructive Testing, Millimeter Waves, Reflectometers

PACS: 81.70.Ex, 07.57.-c

\section{INTRODUCTION}

The Space Shuttle Columbia's catastrophic failure has been attributed to a piece of external tank spray on foam insulation (SOFI) striking the left wing of the orbiter, causing significant damage to some leading edge wing panels [1]. Subsequently, several nondestructive testing (NDT) techniques have been considered for testing the external tank [2-4]. One such technique involves using millimeter waves, which have been shown to easily penetrate the foam and provide high-resolution images of its interior structures [3, 5-10]. In the recent years, continuous-wave (CW) microwave and millimeter wave imaging systems have been effectively used to demonstrate their utility for producing high-resolution images of SOFI-covered metal structures such as the Space Shuttle external fuel tank. Several methods and techniques have been developed or modified for this purpose including the following:

- near-field methods and techniques using small horn antennas [5-6],

- phase and/or magnitude sensitive reflectometers $[3,5-7]$,

- millimeter wave lens-focused imaging systems $[3,6-7]$, 
- synthetic aperture focusing techniques (SAFT) and holography [8],

These methods and techniques have produced high-resolution images at different places of the structure including opening stringers, flange and bolts regions and can easily interrogate thin as well as thick SOFI ( $>9$ ") enabling detection and evaluation of flaws such as:

- voids of different shapes and sizes $[3,5,6,8,9]$,

- disbonds between the metal structures and the SOFI $[3,5,6,8]$,

- delaminations inside the SOFI $[3,5,6,8]$,

- vertical cracks in the SOFI [7].

In addition, these systems have shown great potential for inspecting acreage heat tiles of the orbiter including corrosion of metal substrate under the heat tiles [9].

The millimeter wave circuit portion of these inspection systems are relatively simple, inexpensive, easy-to-use, require little to no signal processing of the raw data, onsite friendly, real-time and small. However, for some specific structures unwanted indications such as a certain interference pattern may be superimposed on the produced images. There are methods by which the influence of this unwanted interference can be reduced, such as the incorporation of an incident angle and the proper use of signal polarization. This paper presents the basics of this problem and describes the use of the methods for reducing this unwanted influence through specific examples.

\section{PROBLEM STATEMENT}

Generally, these unwanted indications occur when a SOFI region with complex geometry including ramps, tilted or curved substrates and stringers is irradiated by an antenna possessing a complex field distribution on the irradiated area. Therefore, there may exist more than one reflected signal from inspected area (e.g., from top of the SOFI and the substrate). Consequently, these signals combine coherently and produce an interference pattern artifact on the produced image. It is well-known that in the case of two signals of equal amplitudes, the total intensity of dc signal component, $I$, at the detector is:

$$
I=2 I_{0}(1+\cos \Delta \varphi),
$$

where $I_{0}$ is the intensity of each signal and $\Delta \varphi$ is the phase difference between the two signals at the detector. It is important to note that in general $I_{0}$ is a complex function dependent on the antenna pattern field distribution. The phase difference is then determined by the difference in propagation distances, $\Delta z$, as $\Delta \varphi=2 \pi \Delta z / \lambda$ ( $\lambda$ is wavelength). The propagation distance difference of $\lambda 2$ and $\lambda$ produces minimum and maximum total intensity, respectively, and at a further increasing of the propagation distance an interference pattern can be generated.

Based on the phase difference between these two signals unwanted indications can change dramatically when the sample is scanned resulting in artifacts (e.g., interference pattern) that can severely reduce the amount of information conveyed by an image. For instance, a horizontal spatial shift, $\Delta x$, of the antenna produces a propagation distance of $\Delta z=\Delta x(\sin \beta)$ where $\beta$ is the tilt angle associated with the relative substrate orientation. The severity may be significant enough so that image processing algorithms may not be able to recover the needed information. It is very attractive to remove or reduce an influence of these unwanted indications at the measurement or hardware level so that either flaws can be detected and evaluated in the raw data or indications of flaws may be detected in the image and may be enhanced employing localized image processing 
algorithms. Thus, the goal of this investigation is to reduce or remove these unwanted indications by manipulating various measurement parameters.

\section{DESCRIPTION OF THE MEASUREMENT APPROACH AND SAMPLES}

To this end, several millimeter wave reflectometers, operating in a wide range of frequencies from $24 \mathrm{GHz}$ to $150 \mathrm{GHz}$, were designed and tested on various SOFI samples and panels. These reflectometers were composed of a mono-static millimeter wave system so that one antenna transmits the signal to the sample and receives the signal reflected from the sample as well. Consequently, the transmitted and received signals correspond to the same illuminated areas/volumes and have identical polarizations. Small horn antennas as well as dielectric lens antennas were incorporated into these systems for obtaining small inspection footprints and hence finer spatial resolutions. Mono-static reflectometers can also produce images that are either phase and/or magnitude sensitive. This is important since depending on the dielectric properties of the structure under inspection and the type of anomaly that may exist in the structure the phase or the magnitude of the reflected signal from an anomaly may be a better indicator of its presence and properties [10]. Finally, when inspecting sample regions with complex structures (e.g., stringers, flanges and bolts), images were produced using proper incident angle and signal polarizations. Polarization diversity is a significant attribute of these millimeter wave measurements. In particular, when using an oblique incident angle the choice of polarization can significantly influence the outcome of the measurements due to the fact that at the oblique incident angles Fresnel reflection coefficient is polarization dependent [11]. In this investigation the samples with intentionally tilted substrates and two distinct polarizations of incident signal were used; namely, one in which the incident electric field polarization vector was perpendicular to the plane of incidence (referred to as the perpendicular polarization) and other in which the incident electric field polarization vector was parallel to the plane of incidence (referred to as the parallel polarization) [13]. In the former case electric field vector was always parallel to the substrate while in the later case there was an angel between this vector and the substrate. It should be noted that definition of the incident angle and the polarization were given for the plane-wave approximation which could be applied to the far-field region of the antennas such as horn antennas and to the focal plane (distance) of focusing lens antennas.

A sample under test was placed on a computer-controlled two-dimensional scanning table with the millimeter wave system held at a certain distance above the sample. Subsequently, as the sample is scanned, the system output voltage is used to produce a raster scan. The measured output voltages are then normalized and assigned different grayscale levels producing a raster "image". It is important to note that by normalizing the images, the grayscale levels in one image do not necessarily correspond to the same output voltage levels in another image.

This measurement approach was also used to measure field distribution (footprints) of the horn antennas and lens antennas used in these investigation. For this purpose a small target (e.g., a rubber patch of $0.5 \mathrm{~mm}$ by $0.5 \mathrm{~mm}$ by $0.5 \mathrm{~mm}$ ) was located on a flat metal substrate and the images of this target were produced using horn and lens antennas at different frequencies and distances from the antennas. These images can be considered as maps of the field distributions of the antennas on the plate since the small target does not disturb their field distributions. For example, Figure 1 shows the measured field distribution for the small horn antenna used in this investigation at different distances, $\mathrm{L}$, between the plate and the antenna aperture in its near-field region at $36 \mathrm{GHz}$. In this case $\mathrm{L}$ $\angle \mathrm{R}$ where $\mathrm{R}=2 \mathrm{D} / \lambda^{2}=217 \mathrm{~mm}$ which corresponds to the far-field distance of this horn antenna with an aperture diagonal dimension of $\mathrm{D}=30 \mathrm{~mm}$. It can be seen from Figure 1 that for this small horn antenna, as the distance between the horn antenna and the sample 
increases, the field distribution changes and hence parameters such as the spatial resolution and the sidelobe interference influence will be changed too, as expected.

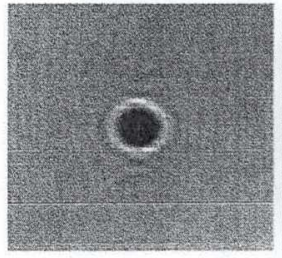

(a)

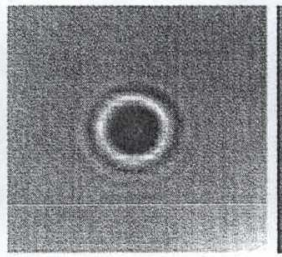

(b)

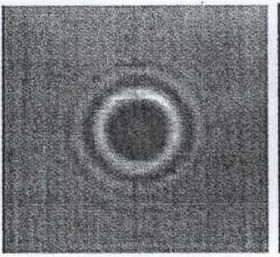

(c)

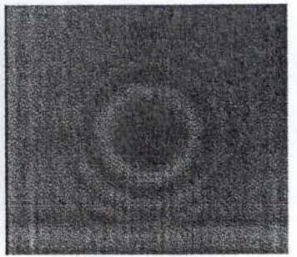

(d)

FIGURE 1. The 36-GHz field distribution (footprints) on the metal plate for the horn antenna at different L/R: (a) 0.2 , (b) 0.4 , (c) 0.6 and (d) 0.8 .

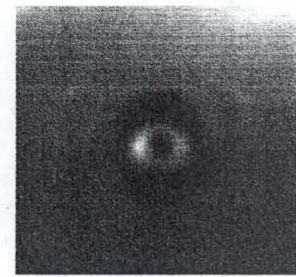

(a)

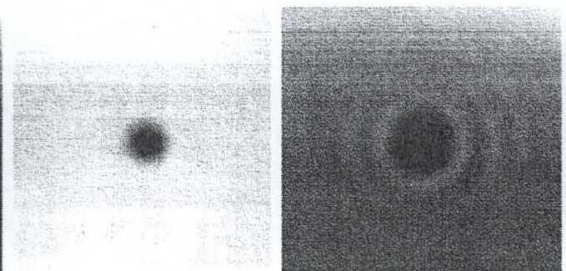

(b) (c)

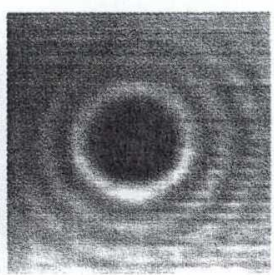

(d)

FIGURE 2. The 100-GHz field distribution (footprints) on the metal plate for the lens antenna at different distances between the lens and the plate: (a) $229 \mathrm{~mm}$, (b) $254 \mathrm{~mm}$ (focal distance), (c) $280 \mathrm{~mm}$, and (d) 305 $\mathrm{mm}$.

The measured field distribution for the $100 \mathrm{GHz}$ lens antenna at four distances in and near its depth-of-field (focal range) is shown in Figure 2. Figure 2 indicates that at the focal distance the field (beam) is quite focused with minimal sidelobes, while more significant sidelobes exist outside of the focal range. It should be noted that this is the lens antenna with short depth-of-field of $\sim 30 \mathrm{~mm}$. Another $100 \mathrm{GHz}$ lens antenna that was also used had a long depth-of-field $(\sim 200 \mathrm{~mm})$ and different field distribution than the short depthof-field lens, in particular the sidelobe levels for the former lens were lower than the latter lens.

Two SOFI samples were used in this investigation. One of the samples was a SOFI wedge sample with several localized anomalies (i.e., voids) of different sizes and at different depths embedded within the SOFI at about $25 \mathrm{~mm}$ above aluminum substrate. Figure 3a shows the schematic of this sample showing (by dashed lines) the area that was imaged. In this investigation the sample was used to provide tilted substrate with conjunction with the SOFI ramp. One of the example of the tilted substrate is shown in Figure $3 b$ where a tilt angle of $\beta=22^{\circ}$ existed between the substrate and the normal to the substrate. Another thick SOFI sample, with substrate dimensions of $550 \mathrm{~mm}$ by $550 \mathrm{~mm}$ and $203 \mathrm{~mm}$ thick was also used in this investigation. However, in this case the aluminum substrate was relatively thin aluminum. As a result of the relatively thick foam on this thin substrate, the four corners of the substrate were lifted up after the spraying of the foam. Consequently, the substrate had an approximately concave shape ("bowl shape"), as shown in Figure 4a. This sample represents a subtly curved substrate with thick SOFI section. Two sets of flaws composed of voids and disbonds with different sizes and shapes were embedded in this panel. One set was placed directly on top of the substrate. Figure $4 \mathrm{~b}$ shows the schematic of these embedded flaws, indicating their sizes and shapes. 


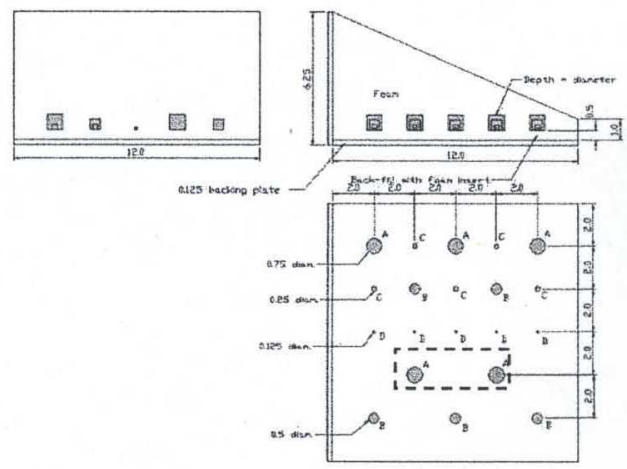

(a)



(b)

FIGURE 3. (a) Schematic of the SOFI wedge sample with embedded voids and (b) the picture of the tilted SOFI wedge sample.

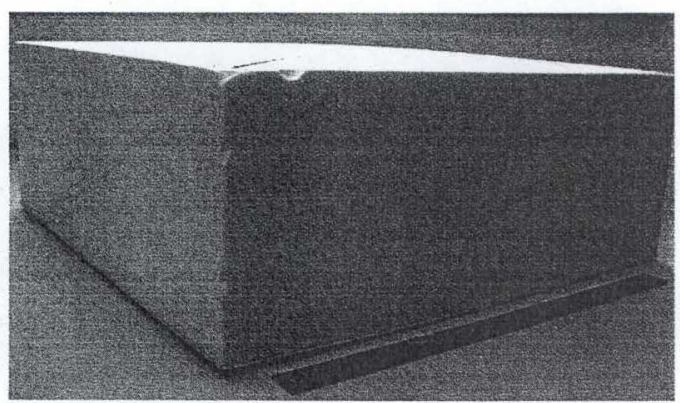

(a)

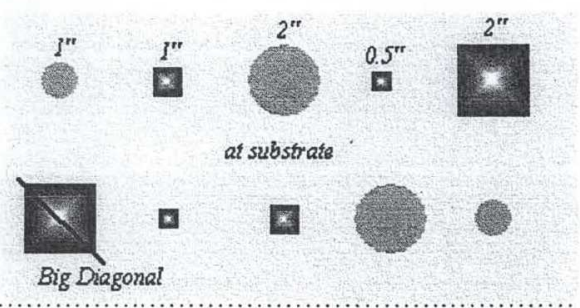

(b)

FIGURE 4. (a) Picture of the thick SOFI wedge sample with a curve substrate and (b) schematic of a half of the sample with voids and disbonds at the substrate.

\section{RESULTS}

\section{Tilted SOFI wedge sample}

Figure 5a shows the image of a portion of the SOFI wedge sample (marked in Figure 3a with dashed lines) with indications of two voids produced using a small horn antenna at $36 \mathrm{GHz}$. The relative position of the sample in this case was that shown in Figure $3 \mathrm{~b}$. The image not only shows the two voids it also shows a series of dark and bright lines (interference pattern). The distance between adjacent dark (bright) lines is determined to be $\sim 11 \mathrm{~mm}$. This distance related to the corresponding horn antenna horizontal shift, $\Delta \mathrm{x}$. A simple calculation at given $\Delta \mathrm{x}=11 \mathrm{~mm}, \lambda=8.3 \mathrm{~mm}$ and $\beta=22^{\circ}$ yields the difference in propagation distances $\Delta \mathrm{z}=4.15 \mathrm{~mm}=\mathrm{N} 2$. It means that the interference pattern amplitude changes as a function of substrate distance to the horn, and this interference pattern is primarily due to the reflections from antenna aperture, top of the SOFI, substrate, sidelobe levels and the influence of incidence angle on reflections from sidelobes. Subsequently, the image was processed using a notch filter capable of removing periodic feature and of significantly reducing the interference pattern effect as shown in the processed image in Figure 5b. The amplitude of reflected signal from the substrate changes across the scan and this fact is evidenced in Figure $5 \mathrm{a}$ as the intensity of the interference lines increases from left to right. In addition, the influence of sidelobes is also evident on the images of the voids in Figure $5 b$. 


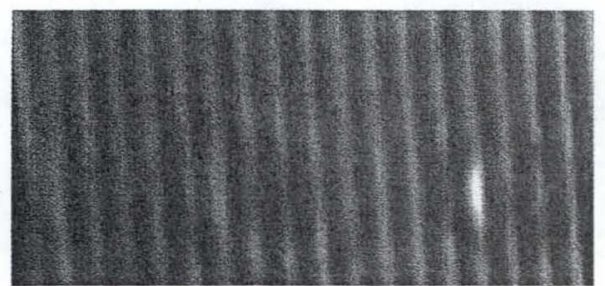

(a)

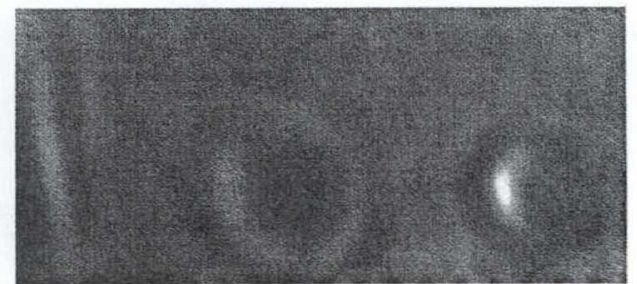

(b)

FIGURE 5. (a) Raw and (b) processed images of the portion ( $200 \mathrm{~mm}$ by $90 \mathrm{~mm}$ ) of the tilted SOFI wedge sample obtained with a small horn antenna at $36 \mathrm{GHz}$.

It should be noted that only the largest voids in the SOFI wedge sample at different positions were detected using the small horn antenna. Better results were obtained with lens antennas at higher frequencies. For instance, Figure 6 shows image of the entire not tilted (i.e., substrate orientation orthogonal to the direction of propagation) SOFI wedge sample obtained at $100 \mathrm{GHz}$ using the lens antenna with the short depth-of-field when the lens was focused at the substrate (Figure 6a) and $64 \mathrm{~mm}$ above the substrate (Figure 6b), respectively. The results show that, with the exception of a few voids the majority of the voids are clearly detected. In addition, the relative size and shapes of the voids are also clearly shown in the images. These images also show additional several small indications that are most likely due to the presence of excess adhesive during the manufacturing process. The most important that the interference pattern is significantly reduced since in this case the reflection from the substrate is constant while that from the SOFI surface (which is much weaker) changes across the scan resulting in gradual change of the intensity in the images from left to right.

To demonstrate the influence of the polarization, Figures 7 shows the images of a slightly tilted $\left(\beta \sim 3^{\circ}\right)$ SOFI wedge sample at $100 \mathrm{GHz}$ using the lens antenna focused at the substrate at two different polarizations. The results show: a) the increased interference pattern when compared to the results in Figure 6, and b) the influence of the interference pattern is not uniform as a function of polarization (i.e., Fresnel reflection coefficient), as stated earlier. Thus, polarization can be used to minimize this undesired effect. Although the images look very similar, the results show that some standoff distance variation can be tolerated without significant degradation in the image quality.

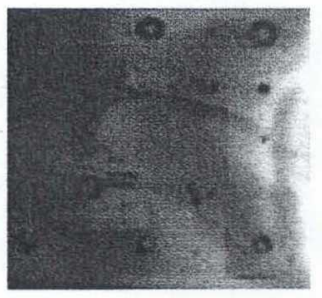

(a)

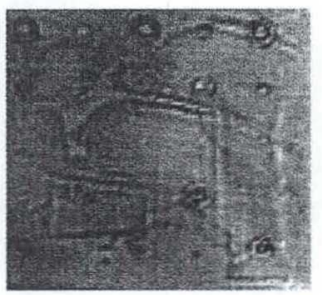

(b)

FIGURE 6. 100-GHz images of the SOFI wedge sample obtained with short depth of focus lens antenna focused at (a) the substrate and (b) $65 \mathrm{~mm}$ above the substrate. 


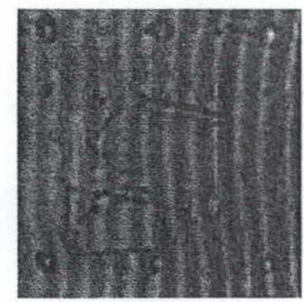

(a)



(b)

FIGURE 7. 100-GHz images of the slightly tilted SOFI wedge sample obtained with the lens antenna focused at the substrate at the (a) perpendicular and (b) parallel polarization.

\section{Thick SOFI sample with curve substrate}

As mentioned earlier, one can see the "bowl shape" of the substrate for the relatively thick SOFI sample, which also had a relatively thin substrate (as shown in Figure 4a). Figure 8 shows the images of the half of this sample with $100 \mathrm{GHz}$ lens antennas focused at the middle of thickness of the sample. The image obtained with the short depth-of-field lens antenna (Figure 8a) shows interference pattern that are relatively strong, but still do not totally mask the indications of embedded voids (placed at the substrate). It is clearly seen in this image that the distance between dark and bright lines are not equal in each image (evidence of the curve substrate). Figure $8 \mathrm{~b}$ shows the image of the same portion of the sample obtained with the long depth-of-field lens antenna. From this image it is clearly seen that the influence of the interference pattern is significantly reduced, while the voids are still detected with ample individual features showing. These results clearly demonstrate the fact that existence of interference pattern depends on the field distribution of lens antenna (e.g., the sidelobe structure and relative intensity).

\section{SUMMARY}

The results can be summarized as follows. Indications of flaws can be masked by unwanted reflections caused by a combination of sources which are:

- complex phase and magnitude field distribution in near-field of radiators (antennas).

- sidelobes and complex field distribution in the different cross sections of the lens-focused electromagnetic beam outside of its focal range.

- reflection signals from the SOFI and substrate of complex geometry (stringers, flanges, bolts, etc.), and their interference.

Remedies for removing or reducing unwanted reflections/indications/influences can be:

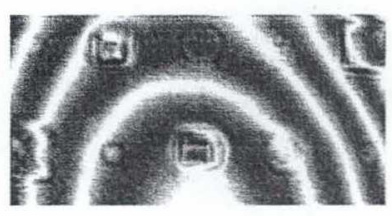

(a)



(b)

FIGURE 8. $100 \mathrm{GHz}$ images of the half thick SOFI sample obtained with (a) short and (b) long depth-offield lens antennas focused at the middle of thickness of the thick SOFI sample. 
- suitable choice of the radiators (i.e., horn and lens antennas) and their performances (e.g., far-field distances for the horn antennas, focus distance and focal range for the lens antennas) and arrangements (e.g., standoff distance, incidence angle).

- use of suitable polarization of the signals for the regions with stringers, flanges, bolts and tilted/curved substrate.

- signal processing.

\section{REFERENCES}

1. "Columbia accident investigation board report," NASA, Aug. 2003.

2. E. Generazio, "The national aeronautics and space administration nondestructive evaluation program for safe and reliable operations," in Review of Progress in QNDE, 25A; edited by D. O. Thompson and D. E. Chimenti, AIP Conference Proceedings vol. 820, American Institute of Physics, Melville, NY (2006), pp. 2-21.

3. R. Zoughi, S. Kharkovsky, and F. Hepburn, "Microwave and millimeter wave testing for the inspection of the space shuttle spray on foam insulation (SOFI) and the acreage heat tiles," in Review of Progress in QNDE, 25A, edited by D. O. Thompson and D. E. Chimenti, AIP Conference Proceedings vol. 820, American Institute of Physics, Melville, NY (2006), pp. 439-446.

4. C.P. Chiou, R. B. Thompson, W. P. Winfree, E. I. Madaras and J. Seebo, "Modelling and processing of terahertz imaging in space shuttle external tank foam inspection," in Review of Progress in $Q N D E, 25 \mathrm{~A}$, edited by D. O. Thompson and D. E. Chimenti, AIP Conference Proceedings vol. 820, American Institute of Physics, Melville, NY (2006), pp. 484 491.

5. S. Shrestha, S. Kharkovsky, R. Zoughi and F. L. Hepburn, Materials Evaluation, 63, pp. 339-344 (2005).

6. S. Kharkovsky, F. Hepburn, J. Walker and R. Zoughi, Materials Evaluation, 63, pp. 516-522 (2005).

7. S. Kharkovsky, R. Zoughi and F. Hepburn, "High Resolution Millimeter Wave Detection of Vertical Cracks in the Space Shuttle External Tank Spray-on-Foam Insulation (SOFI)," in Review of Progress in Quantitative Nondestructive Evaluation 26B, AIP Conference Proceedings, edited by D.O. Thompson and D.E. Chimenti, vol.894, American Institute of Physics, Melville, NY (2007), pp. 1065-1070.

8. J.T. Case, J. Robbins, S. Kharkovsky, R. Zoughi and F. Hepburn, "Microwave and Millimeter Wave Imaging of the Space Shuttle External Tank Spray on Foam Insulation (SOFI) Using Synthetic Aperture Focusing Techniques (SAFT)," in Review of Progress in Quantitative Nondestructive Evaluation 25B, edited by D.O. Thompson and D.E. Chimenti, AIP Conference Proceedings, vol.820, American Institute of Physics, Melville, NY (2006), pp. 1546-1553.

9. J.T. Case, S. Kharkovsky, R. Zoughi and F. Hepburn, "High Resolution Millimeter Wave Inspecting of the Orbiter Acreage Heat Tiles of the Space Shuttle", Proc. of the IEEE Instrumentation and Measurement Technology Conference, Warsaw, Poland, \#7694, (2007).

10. R. Zoughi, Microwave Non-destructive Testing and Evaluation, Netherlands, Kluwer Academic Publishers, 2000.

11. D.M. Pozar, Microwave Engineering, second edition, New York, Addison Wesley, 1990. 